\title{
AF-EQUIVALENCE RELATIONS
}

\author{
MATS MOLBERG
}

\begin{abstract}
Let $R=\lim R_{n}$ be the inductive limit of an ascending sequence of étale finite equivalence relations $R_{n}$ on the zero-dimensional space $X$. We prove that $R$ is an AF-equivalence relation.
\end{abstract}

We start by stating the following theorem, giving the appropriate definitions below. (Recall that an equivalence relation is said to be finite if every equivalence class consists of finitely many elements.)

THEOREM 1. Let $R=(R, \mathscr{T})$ be an étale (countable) equivalence relation on the zero-dimensional (second countable, locally compact) space $X$. The following are equivalent:

(i) $R$ is an AF-equivalence relation;

(ii) $R$ is isomorphic to the étale equivalence relation $R_{(V, E)}$ associated to a Bratteli diagram $(V, E)$;

(iii) $R$ is the inductive limit of an ascending sequence of étale finite equivalence relations $\left\{\left(R_{n}, \mathscr{T}_{n}\right)\right\}$, where $R_{n}$ is open in $R_{n+1}$ for every $n$.

Remark 2. The equivalence between (i) and (ii) is proved in [1]. The implication (i) $\Rightarrow$ (iii) is immediate. One would certainly expect that the converse implication is true. However, it is somewhat surprising that a proof of this has not appeared in the literature. It is the goal of this short paper to provide a proof of the implication (iii) $\Rightarrow$ (i). As will be apparent the proof is not completely straightforward, but requires a careful analysis. Establishing the equivalence between (i) and (iii) is very satisfactory, since it highlights the analogy between AF-equivalence relations in the topological setting, with hyperfinite equivalence relations in the Borel and measure-theoretic settings. In fact, one may say that (iii) would be the most "natural" way to define an AF-equivalence relation $R$, since it explicitly exhibits the approximation to $R$ by finite equivalence relations. 


\section{Introduction}

We start by reminding the reader of the basic definitions concerning étale equivalence relations. For details, cf. [1].

An étale equivalence relation, $R$, on the locally compact, second countable space $X$ is the topological groupoid associated to a countable equivalence relation $R=(R, \mathscr{T})$ on $X$, where the locally compact topology $\mathscr{T}$ on $R$ makes the range map, $r: R \longrightarrow X$ defined by $r((x, y))=x$, a local homeomorphism, i.e. for all $(x, y) \in R$ there exists an open neighborhood $\widetilde{U}_{(x, y)}$ of $(x, y)$ such that

(1) $r\left(\widetilde{U}_{(x, y)}\right)$ is open in $X$;

(2) $r: \widetilde{U}_{(x, y)} \longrightarrow r\left(\widetilde{U}_{(x, y)}\right)$ is a homeomorphism. (In particular $r$ is an open map.)

In groupoid language this is known as an $r$-discrete groupoid (see [2]).

It follows from the definition that if $R^{\prime} \subset R$ is an open subequivalence relation, then $R^{\prime}$ is again étale in the relative topology.

We will say that $\widetilde{U}_{(x, y)}$ satisfies the étale condition at $(x, y)$ (with respect to $r$ ) if (1) and (2) holds. We also say that $\widetilde{U}_{(x, y)}$ is an étale neighborhood of $(x, y)$. Clearly, if $X$ is zero-dimensional, i.e. $X$ has a basis of clopen sets, then we may choose $\widetilde{U}_{(x, y)}$ to be a compact open set. There is also a source map, $s: R \longrightarrow X$, where $s((x, y))=y$. Since the inverse map, $(x, y)^{-1}=(y, x)$, is a homeomorphism, the étale condition could equivalently be stated with respect to the source map. Note also that the map $r \times s: R \longrightarrow X \times X$, defined by $r \times s((x, y))=(x, y)$, is continuous, where $X \times X$ has the product topology.

For $x \in X$ we denote the equivalence class of $x$ assosiated to $R$ by $[x]_{R}$, and the number of elements in its equivalence class is denoted by $\sharp[x]_{R}$. By definition $\sharp[x]_{R}$ will be at most countable, and if $\sharp[x]_{R}$ is finite for every $x \in X$, we say that $R$ is a finite equivalence relation.

Let $\Delta=\{(x, x) \mid x \in X\}$ be the diagonal in $X \times X$ (i.e. the unit space of $R$, when regarded as a groupoid). It is a fact (see [1]) that when $(R, \mathscr{T})$ is an étale equivalence relation, then $\Delta$ is clopen in $R$, and the map $(x, x) \longrightarrow x$, from $\Delta$ to $X$, is a homeomorphism.

We say that a subset $A$ of $X$ is $R$-invariant (or $R$-saturated) if $x \in A$ and $(x, y) \in R$ implies $y \in A$.

Definition 1 (Compact étale equivalence relation). An étale equivalence relation $(R, \mathscr{T})$ on the locally compact, second countable space $X$ is a compact étale equivalence relation (abbreviated CEER) if $R \backslash \Delta$ is a compact subset of $R$. 
The following result concerning CEERs holds, cf. [1, Proposition 3.2].

Proposition 2. If $(R, \mathscr{T})$ is a CEER on $X$, then

(i) $\mathscr{T}$ is the relative topology from $X \times X$;

(ii) $R$ is a closed subset of $X \times X$, and the quotient topology of the quotient space $X / R$ is Hausdorff;

(iii) $R$ is uniformly finite (i.e. $\exists N \in \mathrm{N}$ such that $\sharp[x]_{R} \leq N$ for all $x \in X$ ).

Remark 3. Generally, for an equivalence relation $R$ on $X$ to be étale, the relative topology from the product topology on $X \times X$ is too coarse. Also, regarding (iii), there are easy examples of uniformly étale finite equivalence relations not being CEER. (See Example 4 below.)

Example 4. Let $X$ be some Cantor subset of $[0,1]$ containing the point $\frac{1}{2}$ and being symmetric around $\frac{1}{2}$, i.e. $\frac{1}{2}-\epsilon \in X \Leftrightarrow \frac{1}{2}+\epsilon \in X$ for $0 \leq \epsilon \leq \frac{1}{2}$. Such a Cantor set can easily be constructed. Define $R=\Delta \bigcup\{(x, 1-x) \mid x \in$ $X$ \}. Observe that

$$
\sharp[x]_{R}= \begin{cases}1, & \text { if } x=\frac{1}{2} ; \\ 2, & \text { otherwise. }\end{cases}
$$

Giving $R$ the relative topology $\mathscr{T}_{\text {rel }}$ from the product topology on $X \times X$, one sees immediately that the étale condition fails at the point $\left(\frac{1}{2}, \frac{1}{2}\right)$ as there is no open neighborhood making the range map injective. However, this is the only point causing problems, and $R$ is easily made into an étale equivalence relation by making the topology finer. Let $\mathscr{T}$ be the topology generated by $\mathscr{T}_{\text {rel }}$ and $\Delta$. In fact, for any open neighborhood $B \in \mathscr{T}_{\text {rel }}$ of $\left(\frac{1}{2}, \frac{1}{2}\right)$, put $\widetilde{U}_{\left(\frac{1}{2}, \frac{1}{2}\right)}=B \bigcap \Delta$. Now $\widetilde{U}_{\left(\frac{1}{2}, \frac{1}{2}\right)}$ fulfills the étale condition for $\left(\frac{1}{2}, \frac{1}{2}\right)$. See Figure 1 for illustration.

Definition 5 (AF-equivalence relation). Let $\left\{\left(R_{n}, \mathscr{T}_{n}\right)\right\}_{n=1}^{\infty}$ be an ascending sequence of CEERs on a zero-dimensional (second countable, locally compact) space $X$, so that the inclusion map of $R_{n}$ into $R_{n+1}$ is continuous for each $n$. Let $(R, \mathscr{T})$ be the inductive limit of $\left\{\left(R_{n}, \mathscr{T}_{n}\right)\right\}_{n=1}^{\infty}$ with the inductive limit topology $\mathscr{T}$, i.e. $R=\bigcup_{i=1}^{\infty} R_{n}$ and $\widetilde{U} \in \mathscr{T}$ if and only if $\widetilde{U} \cap R_{n} \in \mathscr{T}_{n}$ for every $n$. We say that $(R, \mathscr{T})$ is an AF-equivalence relation on $X$ and we write $(R, \mathscr{T})=\lim \left(R_{n}, \mathscr{T}_{n}\right)$. (In this case one can prove that $R_{n}$ is open in $R_{n+1}$, i.e. $R_{n} \in \mathscr{T}_{n+1}, c f$. [1].)

The following proposition, which is proved in [1, Proposition 3.12], will simplify our proof in the sense that we will be able to focus exclusively on (étale) finite equivalence relations, and show these are AF, in order to prove our result. 


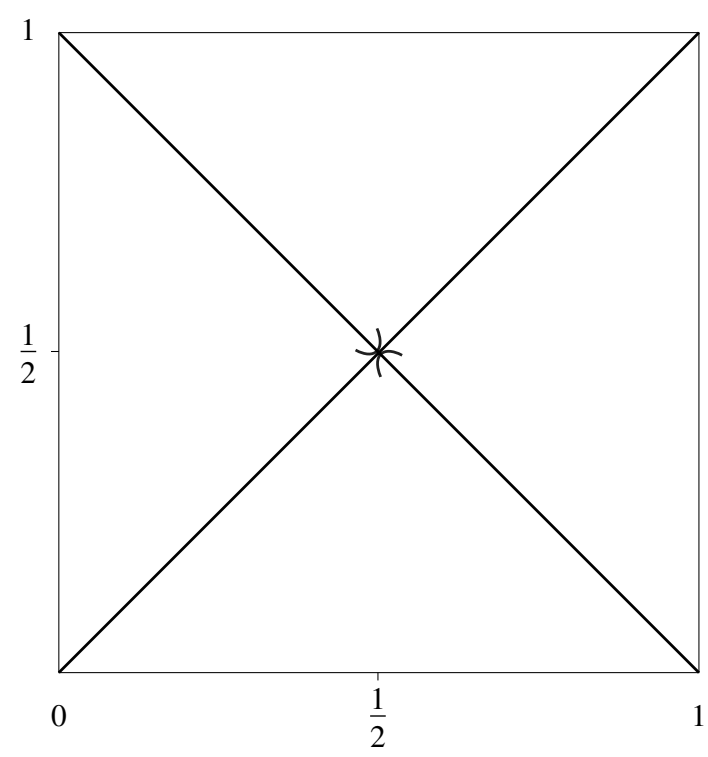

FiguRE 1. "Cantor-cross" equivalence relation.

PROPOSITION 6.

(i) Let $R=\underset{\longrightarrow}{\longrightarrow} R_{n}$ be the inductive limit of an ascending sequence of $A F$ equivalence relations $R_{n}=\left(R_{n}, \mathscr{T}_{n}\right)$ on $X$, such that $R_{n}$ is open in $R_{n+1}$ for each $n$ (which one can show implies that the inclusion map is a homeomorphic embedding of $R_{n}$ into $\left.R_{n+1}\right)$. Then $R=(R, \mathscr{T})$ is an $A F$-equivalence relation on $X$.

(ii) Let $R=(R, \mathscr{T})$ be an AF-equivalence relation on $X$, and let $R^{\prime} \subset R$ be a subequivalence relation which is open, i.e. $R^{\prime} \in \mathscr{T}$. Then $\left(R^{\prime}, \mathscr{T}^{\prime}\right)$ is an $A F$-equivalence relation, where $\mathscr{T}^{\prime}$ is the relative topology.

Example 7 (Example 4 revisited). Going back to $(R, \mathscr{T})$ from Example 4 it is easy to establish that it is not CEER. However, it is an AF-relation. In fact, let $\left\{U_{n}\right\}_{n=1}^{\infty} \subset X$ be a sequence of clopen neighborhoods of $\frac{1}{2}$ satisfying the following

(i) $U_{n}$ is symmetric around $\frac{1}{2}$ for all $n \in \mathrm{N}$;

(ii) $U_{n+1} \subset U_{n}$ for all $n \in \mathrm{N}$;

(iii) $\bigcap_{n=1}^{\infty} U_{n}=\left\{\frac{1}{2}\right\}$.

Define, for all $n \in \mathrm{N}, R_{n}=\Delta \bigcup\left\{(x, 1-x) \mid x \in U_{n}^{c}\right\}$, an let $\mathscr{T}_{n}$ be the relative topology from the product topology on $X \times X$. Figure 2 gives an illustration of $\left(R_{n}, \mathscr{T}_{n}\right)$. Observe that the problem concerning the point $\frac{1}{2}$ in Example 4 


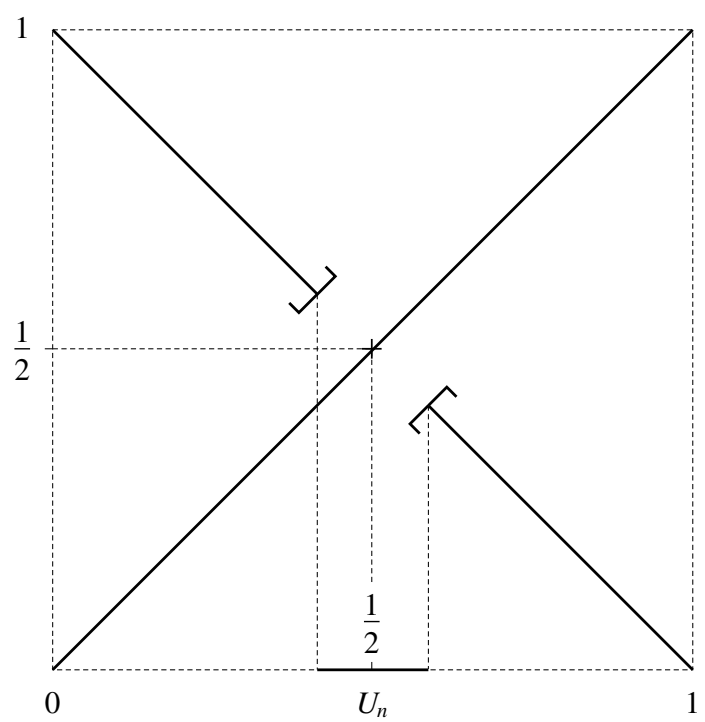

FIGURE 2. CEER sub-equivalence relation of the "Cantorcross" equivalence relation in Figure 1.

is now eliminated. In fact it is easy to show that $\left(R_{n}, \mathscr{T}_{n}\right)$ is CEER for all $n$. Also, it is clear, by the choice of $\left\{U_{n}\right\}_{n=1}^{\infty}$, that $R_{n+1} \subset R_{n}$, and that $R_{n} \in \mathscr{T}_{n+1}$ for all $n \in \mathrm{N}$. Now, $(R, \mathscr{T})=\lim \left(R_{n}, \mathscr{T}_{n}\right)$, and hence $(R, \mathscr{T})$ is an AFequivalence relation. This particular example gives one of the clues of how to prove (iii) $\Rightarrow$ (i) of Theorem 1 .

From this point on $(R, \mathscr{T})$ denotes an étale finite equivalence relation on the zero-dimensional (second countable, locally compact) space $X$. Sometimes the $\mathscr{T}$ will be dropped, and we write $R$ instead of $(R, \mathscr{T})$.

Before getting on with the proof we need to introduce some notation and terminology. For $\widetilde{U}, \widetilde{V} \subset R$, we let

$$
\begin{aligned}
\widetilde{U} \widetilde{V} & =\{(x, y) \mid \exists z,(x, z) \in \widetilde{U} \text { and }(z, y) \in \widetilde{V}\} \\
\widetilde{U}^{-1} & =\{(y, x) \mid(x, y) \in \widetilde{U}\} .
\end{aligned}
$$

For $x \in X$ let $r^{-1}(\{x\})=\left\{\left(x, x_{1}\right),\left(x, x_{2}\right), \ldots,\left(x, x_{k}\right)\right\}$, where $x_{1}, x_{2}, \ldots, x_{k}$ are distinct elements of $X$, and we set $x_{1}=x$. For each pair $\left(x_{i}, x_{j}\right) \in R$, choose a compact open neighborhood $\widetilde{U}_{\left(x_{i}, x_{j}\right)}$ of $\left(x_{i}, x_{j}\right)$ satisfying the following properties.

(1) $\widetilde{U}_{\left(x_{i}, x_{j}\right)}$ satisfies the étale condition at $\left(x_{i}, x_{j}\right), i, j=1, \ldots, k$ with respect to both $r$ and $s$; 
(2) $r\left(\widetilde{U}_{\left(x_{i}, x_{j}\right)}\right) \bigcap r\left(\widetilde{U}_{\left(x_{i^{\prime}}, x_{j^{\prime}}\right)}\right)=\emptyset$ whenever $i \neq i^{\prime}$ and $s\left(\widetilde{U}_{\left(x_{i}, x_{j}\right)}\right) \bigcap s\left(\widetilde{U}_{\left(x_{i^{\prime}}, x_{j^{\prime}}\right)}\right)=\emptyset$ whenever $j \neq j^{\prime}$;

(3) $r\left(\widetilde{U}_{\left(x_{i}, x_{1}\right)}\right)=\ldots=r\left(\widetilde{U}_{\left(x_{i}, x_{k}\right)}\right)=s\left(\widetilde{U}_{\left(x_{1}, x_{i}\right)}\right)=\ldots=s\left(\widetilde{U}_{\left(x_{k}, x_{i}\right)}\right)$ for all $i$;

(4) $\left(\widetilde{U}_{\left(x_{i}, x_{j}\right)}\right)^{-1}=\widetilde{U}_{\left(x_{j}, x_{i}\right)}$ for all $i$ and $j$;

(5) $\widetilde{U}_{\left(x_{i}, x_{j^{\prime}}\right)} \widetilde{U}_{\left(x_{j^{\prime}}, x_{j}\right)}=\widetilde{U}_{\left(x_{i}, x_{j}\right)}$ for all $i, j^{\prime}$ and $j$.

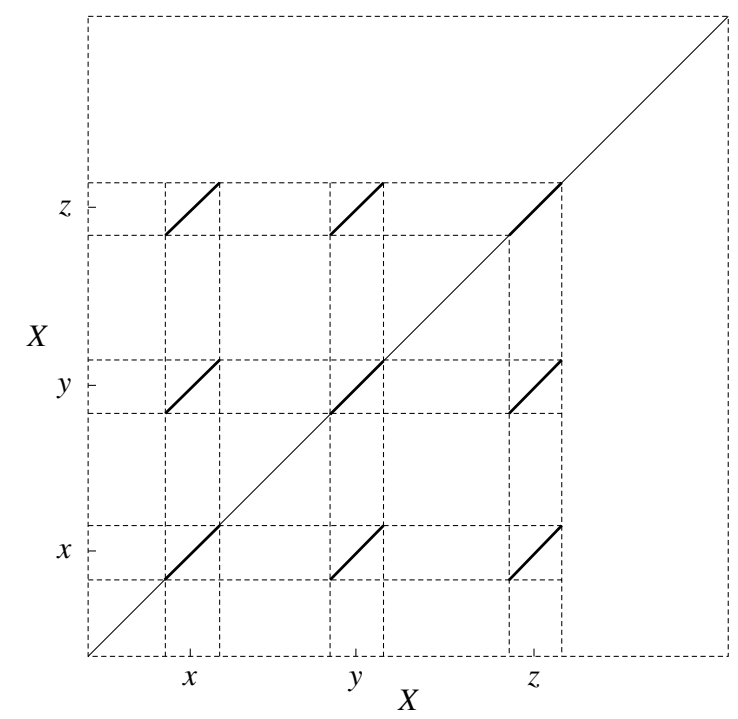

FIGURE 3. Étale collection for $[x]_{R}=\{x, y, z\}$ drawn as bold line segments.

We call the set $\left\{\tilde{U}_{\left(x_{i}, x_{j}\right)}\right\}_{i, j=1}^{k}$ an étale collection (for $[x]=[x]_{R}$ ), and denote it by $\widetilde{U}_{[x]}$. It is easy to see that $\widetilde{U}_{[x]}$ is an equivalence relation contained in $R$. The existence of an étale collection follows in a straightforward way from the étaleness of $R$. We refer to [1] for details. (See Figure 3 for an illustration of an étale collection where $k=3$.) Also, to an étale collection for $[x]=[x]_{R}$, we define the associated étale neighborhood of $[x]$, denoted by $U_{[x]}$, to be the disjoint union (we use “ $\bigsqcup$ ” to denote disjoint union)

$$
\bigsqcup_{i=1}^{k} r\left(\tilde{U}_{\left(x_{i}, x_{j}\right)}\right)=\bigsqcup_{i=1}^{k} U_{x_{i}}=\bigsqcup_{w \in[x]} U_{w}
$$

where $U_{x_{i}}=r\left(\widetilde{U}_{\left(x_{i}, x_{j}\right)}\right)$. (Note that by $(3), r\left(\widetilde{U}_{\left(x_{i}, x_{j}\right)}\right)=r\left(\widetilde{U}_{\left(x_{i}, x_{j^{\prime}}\right)}\right)$ for $j, j^{\prime} \in$ $\{1, \ldots, k\}$.) We may thus suggestively write $U_{[x]}=r\left(\widetilde{U}_{[x]}\right)$. For later use we 
note that if $z \in U_{[x]}$, then $\sharp[z]_{R} \geq \sharp[x]_{R}$, which is an immediate consequence of the definition of an étale collection. Keep in mind that $\widetilde{U}_{[x]}$ is a compact open subset of $R$, and $U_{x_{i}}, i=1, \ldots, k$, and $U_{[x]}$ are compact open subsets of $X$. Furthermore, $\widetilde{U}_{[x]}$, in the relative topology from $R$, is a compact étale equivalence relation on $U_{[x]}$, and so by Proposition 2 (i) the topology on $\widetilde{U}_{[x]}$ is the relative topology from $U_{[x]} \times U_{[x]}$.

\section{Proof of (iii) $\Rightarrow$ (i) of Theorem 1}

We will first assume $X$ to be compact. (The locally compact case will be dealt with afterwards.) By Proposition 6(i) we may assume that $R=(R, \mathscr{T})$ is an étale finite equivalence relation on $X$. We will henceforth denote the $R$-equivalence class $[x]_{R}$ of $x$ by $[x]$.

Fix $x \in X$ and some étale collection $\widetilde{U}_{[x]}$ for $[x]$, and define

$$
R^{\prime}=R_{\widetilde{U}_{[x]}}=\widetilde{U}_{[x]} \bigcup\left(R \bigcap\left(U_{[x]}^{c} \times U_{[x]}^{c}\right)\right)
$$

It is easy to see that $R^{\prime}$ is a subequivalence relation of $R$. We note that $R \bigcap\left(U_{[x]}^{c} \times U_{[x]}^{c}\right)$ is an open subset of $R$, as it is the inverse image of $U_{[x]}^{c} \times U_{[x]}^{c}$ under the continuous map $r \times s$. As a consequence we have that $R^{\prime}$ is an open subequivalence relation of $R$, and hence étale in the relative topology. By construction we get that $U_{[x]}=r\left(\widetilde{U}_{[x]}\right)$ is an $R^{\prime}$-invariant subset of $X$.

Now choose, for all $x \in X$, an étale collection $\widetilde{U}_{[x]}$ of $[x]$. By compactness of $X$, there exists a finite set $\left\{\widetilde{U}_{\left[x^{(1)}\right]}, \ldots, \widetilde{U}_{\left[x^{(m)}\right]}\right\}$ such that $\bigcup_{i=1}^{m} U_{\left[x^{(i)}\right]}=X$. Define

$$
\widetilde{R}=\bigcap_{i=1}^{m} R_{\widetilde{U}_{\left[x^{(i)}\right]}} .
$$

LEMMA 1. $\widetilde{R}=\bigcap_{i=1}^{m} R_{\widetilde{U}_{\left[x^{(i)}\right]}}$ is a compact and open subequivalence relation of $R$.

Proof. Since $\widetilde{R}$ is a finite intersection of open sub-equivalence relations of $R$, we only need to show that $\widetilde{R}$ is compact.

Let $\left\{\left(x_{n}, y_{n}\right)\right\}_{n=1}^{\infty} \subset \widetilde{R}$. Since $X$ is compact we can find a subsequence, which we again will denote by $\left\{\left(x_{n}, y_{n}\right)\right\}_{n=1}^{\infty}$, such that $x_{n} \longrightarrow x$ and $y_{n} \longrightarrow y$ in $X$. We want to show that $\left(x_{n}, y_{n}\right) \longrightarrow(x, y)$ in $\widetilde{R}$, which will prove that $\widetilde{R}$ is compact. Choose an $i \in\{1, \ldots, m\}$, such that $x \in U_{\left[x^{(i)}\right]}$. Then there exists $N \in \mathrm{N}$ such that $\left\{x_{n}\right\}_{n=N}^{\infty} \subset U_{\left[x^{(i)}\right]}$. Since $\widetilde{R} \subset R_{\widetilde{U}_{\left[x^{(i)}\right]}}$ and since $U_{\left[x^{(i)}\right]}$ is $R_{\widetilde{U}_{\left[x^{(i)}\right]}}{ }^{-}$ invariant, we have that $\left\{y_{n}\right\}_{n=N}^{\infty} \subset U_{\left[x^{(i)}\right]}$. Furthermore, $U_{\left[x^{(i)}\right]}$ is compact, so $y \in U_{\left[x_{i}\right]}$. Since $\widetilde{U}_{\left[x^{(i)}\right]}$ has the relative topology from $U_{\left[x^{(i)}\right]} \times U_{\left[x^{(i)}\right]}$, this implies that $\left(x_{n}, y_{n}\right) \longrightarrow(x, y)$ in $R_{\widetilde{U}_{\left[x^{(i)}\right]}}$, and hence in $R$. 
We now need to show that $(x, y) \in \widetilde{R}$, or, equivalently, $(x, y) \in R_{\widetilde{U}_{\left[x^{(j)}\right]}}$ for all $j=1, \ldots, k$. Fix $j$. Then, since $U_{\left[x^{(j)}\right]}$ is $R_{\widetilde{U}_{\left[x^{(j)}\right]}}$-invariant, the only two possibilities are that $x, y \in U_{\left[x^{(j)}\right]}$, or $x, y \in U_{\left[x^{(j)}\right]}^{c}$. If $x, y \in U_{\left[x^{(j)}\right]}$, then, by the above argument, $(x, y) \in R_{\widetilde{U}_{\left[x^{(j)}\right.}}$. If $x, y \in U_{\left[x^{(j)}\right]}^{c}$, then certainly $(x, y) \in$ $U_{\left[x^{(j)}\right]}^{c} \times U_{\left[x^{(j)}\right]}^{c}$. We know already that $(x, y) \in R$, and so $(x, y) \in R_{\widetilde{U}_{\left[x^{(j)}\right.}}$. This finishes the proof of the lemma.

We introduce some terminology. Let $d$ be some metric on $X$ compatible with the topology of $X$. Let $x \in X$, with $[x]=\left\{x_{1}, \ldots, x_{k}\right\}$, and let $\widetilde{U}_{[x]}=$ $\left\{\widetilde{U}_{\left(x_{i}, x_{j}\right)}\right\}_{i, j=1}^{k}$ be some étale collection for $[x]$. Then we define $\left|U_{[x]}\right|$ (called the diameter of $U_{[x]}=\bigsqcup_{i=1}^{k} U_{x_{i}}$ ) to be the maximum of the diameters of $U_{x_{i}}=r\left(\widetilde{U}_{\left(x_{i}, x_{j}\right)}\right), i=1, \ldots, k$, that is

$$
\left|U_{[x]}\right|=\max \left\{\operatorname{diameter}\left(U_{x_{i}}\right) \mid i=1, \ldots, k\right\} .
$$

Now let a finite number of étale collections $\left\{\widetilde{U}_{\left[x^{(i)}\right]} \mid i=1, \ldots, m\right\}$ be given such that $\bigcup_{i=1}^{m} U_{\left[x^{(i)}\right]}=X$. We say that $\tilde{\mathscr{U}}=\left\{\widetilde{U}_{\left[x^{(i)}\right]} \mid i=1, \ldots, m\right\}$ is a full set of étale collections and that $R_{\widetilde{U}}=\bigcap_{i=1}^{m} R_{\widetilde{U}_{\left[x^{(i)}\right]}}$ is the CEER associated to $\widetilde{\mathscr{U}}$. We define the diameter of $\tilde{\mathcal{U}}$, denoted $|\widetilde{\mathscr{U}}|$, to be $\max \left\{\left|U_{\left[x^{(i)}\right]}\right| \mid i=1, \ldots, m\right\}$.

We want to construct a nested sequence

$$
R_{1} \subset R_{2} \subset R_{3} \subset \cdots \subset R
$$

of compact and open subequivalence relations of $R$, such that $R=\bigcup_{n=1}^{\infty} R_{n}$. Then clearly

$$
(R, \mathscr{T})=\underline{\lim }\left(R_{n}, \mathscr{T}_{n}\right)
$$

where $\mathscr{T}_{n}$ denotes the relative topology on $R_{n}$, and so $R=(R, \mathscr{T})$ is an AFequivalence relation on $X$. This will finish the proof for the case where $X$ is compact.

Assume we have constructed

$$
R_{1} \subset R_{2} \subset \cdots \subset R_{n} \subset R
$$

where each $R_{k}=R_{\widetilde{U}_{k}}$ is a compact and open subequivalence relation of $R$ associated to some full set $\tilde{U}_{k}$ of étale collections, and such that the diameter of $\widetilde{\mathscr{U}}_{k}$ is less than $\frac{1}{k}$, i.e $\left|\widetilde{\mathscr{U}}_{k}\right|<\frac{1}{k}$. Using Lemma 1 we can clearly construct $R_{1}$ with these properties. So assume $n>1$. We want to construct $R_{n \pm 1}$ such that $R_{\widetilde{\mathscr{U}}_{n}}=R_{n} \subset R_{n+1} \subset R$, and $R_{n+1}$ to be associated to a full set $\widetilde{U}_{n+1}$ of étale collections, i.e $R_{n+1}=R_{\widetilde{U}_{n+1}}$, such that $\left|\tilde{U}_{n+1}\right|<\frac{1}{n+1}$.

Let $\widetilde{\mathscr{U}}_{n}=\left\{\widetilde{U}_{\left[x^{(i)}\right]} \mid i=1, \ldots, m_{n}\right\}$. For every $y \in X$ pick an étale collection $\widetilde{U}_{[y]} \subset R$ for $[y]$ such that 
(i) $\left|U_{[y]}\right|<\frac{1}{n+1}$.

(ii) If $z, w \in[y]$ and $(z, w) \in \widetilde{U}_{\left(x_{k}^{(i)}, x_{l}^{(i)}\right)}$, where $\widetilde{U}_{\left(x_{k}^{(i)}, x_{l}^{(i)}\right)} \in \widetilde{U}_{\widetilde{U}_{\left[x^{(i)}\right]}}$ for some $i=1, \ldots, m_{n}$, then $\widetilde{U}_{(z, w)} \subset \widetilde{U}_{\left(x_{k}^{(i)}, x_{l}^{(i)}\right)}$, where $\widetilde{U}_{(z, w)} \in \widetilde{U}_{[y]}$.

The existence of $\widetilde{U}_{[y]}$ satisfying (i) and (ii) follows easily from the étaleness of $R$.

Now choose a full set of étale collections $\tilde{U}_{n+1}=\left\{\widetilde{U}_{\left[y^{(i)}\right]} \mid i=1, \ldots, m_{n+1}\right\}$ such that every $\widetilde{U}_{\left[y^{(i)}\right]}$ satisfies (i) and (ii), and let $R_{n+1}=R_{\tilde{U}_{n+1}}$ be the compact and open subequivalence relation of $R$ associated to $\tilde{\mathscr{U}}_{n+1}$. (In the sequel we use $x$ 's and $y$ 's when we refer to sets associated to $\widetilde{\mathscr{U}}_{n}$ and $\widetilde{\mathscr{U}}_{n+1}$, respectively, in order to facilitate the reading.) We claim that $R_{n} \subset R_{n+1}$. In fact, let $\left(x, x^{\prime}\right) \in R_{n}$. Suppose $x \in U_{\left[y^{(i)}\right]}$ for some $i \in\left\{1, \ldots, m_{n+1}\right\}$. Now $U_{\left[y^{(i)}\right]}=\bigsqcup_{\tilde{y} \in\left[y^{(i)}\right]} U_{\tilde{y}}$, and so $x \in U_{\tilde{y}}$ for some $\tilde{y} \in\left[y^{(i)}\right]$. There exists some $j \in\left\{1, \ldots, m_{n}\right\}$ such that

$$
\tilde{y} \in U_{\left[x^{(j)}\right]}=r\left(\widetilde{U}_{\left[x^{(j)}\right]}\right)=\bigsqcup_{\tilde{x} \in\left[x^{(j)}\right]} U_{\widetilde{x}} .
$$

Hence $(\widetilde{y}, \widetilde{y}) \in \widetilde{U}_{(\widetilde{x}, \widetilde{x})}$ for some $\widetilde{x} \in\left[x^{(j)}\right]$. By condition (ii) above we get that $\widetilde{U}_{(\tilde{y}, \tilde{y})} \subset \widetilde{U}_{(\widetilde{x}, \widetilde{x})}$, and so

$$
U_{\widetilde{y}}=r\left(\widetilde{U}_{(\widetilde{y}, \widetilde{y})}\right) \subset r\left(\widetilde{U}_{(\widetilde{x}, \widetilde{x})}\right)=U_{\widetilde{x}} \subset U_{\left[x^{(j)}\right]} .
$$

Since $x \in U_{\tilde{y}}$, we get that $x \in U_{\tilde{x}}$. Hence there exists $\widetilde{\widetilde{x}} \in\left[x^{(j)}\right]$ such that $\left(x, x^{\prime}\right) \in \widetilde{U}_{(\widetilde{x}, \widetilde{\tilde{x}})}$, since $\left(x, x^{\prime}\right) \in R_{\widetilde{U}_{\left[x^{(j)}\right]}}$. Also, since $\widetilde{y} \in U_{\widetilde{y}} \subset U_{\widetilde{x}}$, we get that $(\tilde{y}, \widetilde{\widetilde{y}}) \in \widetilde{U}_{(\widetilde{x}, \widetilde{x})}$ for some $\widetilde{\tilde{y}} \in[\widetilde{y}]=\left[y^{(i)}\right]$. By condition (ii), $\widetilde{U}_{(\tilde{y}, \widetilde{y})} \subset \widetilde{U}_{(\widetilde{x}, \widetilde{x})}$. Since $x \in U_{\tilde{y}}$ and $\left(x, x^{\prime}\right) \in \widetilde{U}_{(\widetilde{x}, \widetilde{x})}$, étaleness of $\widetilde{U}_{(\widetilde{x}, \widetilde{x})}$ implies that $\left(x, x^{\prime}\right) \in$ $\widetilde{U}_{(\tilde{y}, \widetilde{y})}$. So $\left(x, x^{\prime}\right) \in \widetilde{U}_{\left[y^{(i)}\right]} \subset R_{\widetilde{U}_{\left[y^{(i)}\right]}}$.

If we had assumed at the start that $x \notin U_{\left[y^{(i)}\right]}$, then our argument would yield that also $x^{\prime} \notin U_{\left[y^{(i)}\right]}$, and so $\left(x, x^{\prime}\right) \in R \bigcap\left(U_{\left[y^{(i)}\right]}^{c} \times U_{\left[y^{(i)}\right]}^{c}\right) \subset R_{\widetilde{U}_{\left[y^{(i)}\right]}}$. We conclude that $\left(x, x^{\prime}\right) \in R_{n+1}=R_{\widetilde{U}_{n+1}}=\bigcap_{i=1}^{m_{n+1}} R_{\widetilde{U}_{\left[y^{(i)}\right]}}$.

We now prove that $R=\bigcup_{n=1}^{\infty} R_{n}$. Let $(x, y) \in R$, and let $\widetilde{V}_{[x]} \subset R$ be an étale collection for [x], with $V_{[x]}=r\left(\widetilde{V}_{[x]}\right)$. Then $x \in V_{x}$, where $V_{[x]}=$ $\bigsqcup_{w \in[x]} V_{w}$. Let $\epsilon=\operatorname{distance}\left(x, V_{x}^{c}\right)$. Then $\epsilon>0$ since $x \in V_{x}$ and $V_{x}$ is clopen. Choose $N$ so large that $\frac{1}{N}<\epsilon$. Now assume $x \in U_{\left[x^{(i)}\right]}=r\left(\widetilde{U}_{\left[x^{(i)}\right]}\right)$ for some étale collection $\widetilde{U}_{\left[x^{(i)}\right]} \in \widetilde{\mathscr{U}}_{N}, i \in\left\{1, \ldots, m_{N}\right\}$, where $R_{N}=R_{\widetilde{U}_{N}}$. Then $x \in U_{\widetilde{x}}$ for some $\tilde{x} \in\left[x^{(i)}\right]$, where $U_{\left[x^{(i)}\right]}=\bigsqcup_{z \in\left[x^{(i)}\right]} U_{z}$. Since diameter $\left(U_{\widetilde{x}}\right)<$ $\frac{1}{N}$, we get that $U_{\tilde{x}} \subset V_{x}$. In particular, $\widetilde{x} \in V_{[x]}$. Since both $\widetilde{V}_{[x]}$ and $\widetilde{U}_{\left[x^{(i)}\right]}$ are étale collections, and $x, \tilde{x} \in V_{[x]} \cap U_{\left[x^{(i)}\right]}$, it follows that $\sharp[x]=\sharp[\tilde{x}]=\sharp\left[x^{(i)}\right]$. 
(Cf. the observation we made when we introduced the definition of an étale collection.) This, combined with $x \in U_{\widetilde{x}} \subset V_{x}$, implies that $r^{-1}(\{x\}) \subset \widetilde{U}_{\left[x^{(i)}\right]}$, and so $(x, y) \in \widetilde{U}_{\left[x^{(i)}\right]}$.

If we had assumed that $x \notin U_{\left[x^{(i)}\right]}$, the above argument would yield that $y \notin U_{\left[x^{(i)}\right]}$. In both cases we may conclude that $(x, y) \in R_{\widetilde{U}_{\left[x^{(i)}\right]}}$. This proves that $(x, y) \in R_{N}=R_{\widetilde{U}_{N}}$, and thus the proof is complete for $X$ compact.

If $X$ is locally compact, there exist a sequence of compact and open subsets $\left\{V_{n}\right\}_{n=1}^{\infty}$ of $X$, such that $X=\bigcup_{n=1}^{\infty} V_{n}$ and $V_{n} \subset V_{n+1}$ for every $n$. Let $R_{n}$ be the subequivalence relation of $R$ defined by

$$
R_{n}=\left\{R \bigcap\left(V_{n} \times V_{n}\right)\right\} \bigcup \Delta
$$

where $\Delta$ is the diagonal of $X \times X$. Since $R \bigcap\left(V_{n} \times V_{n}\right)$ is the inverse image of $V_{n} \times V_{n}$ under the continuous map $r \times s$, we get that $R_{n}$ is open in $R$, and so $R_{n}$, in the relative topology, is an étale equivalence relation on $X$. Clearly

$$
R_{1} \subset R_{2} \subset \cdots \subset R_{n} \subset R_{n+1} \subset \cdots \subset R=\bigcup_{n=1}^{\infty} R_{n}
$$

and so $R$ is the inductive limit of $\left\{R_{n}\right\}_{n=1}^{\infty}$, i.e. $R=\lim _{\longrightarrow} R_{n}$. Since $V_{n}$ is compact, and $R \bigcap\left(V_{n} \times V_{n}\right)$ clearly is an étale finite equivalence relation on $V_{n}$, we get by our result above that $R \bigcap\left(V_{n} \times V_{n}\right)$ is an AF-equivalence relation on $V_{n}$. This implies that $R_{n}$ is an AF-equivalence relation on $X$. By Proposition 6 we conclude that $R$ is an AF-equivalence relation. This completes the proof of (iii) $\Rightarrow$ (i) of Theorem 1 .

AcKnowledgement. The author would like to thank Ian Putnam for helpful comments and suggestions.

\section{REFERENCES}

1. Giordano, T., Putnam, I., Skau, C., Affable equivalence relations and orbit structure of Cantor dynamical systems, Ergodic Theory Dynam. Systems 24 (2004), no. 2, 441-475.

2. Renault, J., A Groupoid Approach to $C^{*}$-algebras, Lecture Notes in Math. 739 (1980).

INSTITUTT FOR MATEMATISKE FAG

NTNU

NO-7491 TRONDHEIM

NORWAY

E-mail: Mats.Molberg@hihm.no 